\title{
A Viscosity Approximation Method for Finding Common Solutions of Variational Inclusions, Equilibrium Problems, and Fixed Point Problems in Hilbert Spaces
}

\author{
Somyot Plubtieng ${ }^{1,2}$ and Wanna Sriprad ${ }^{1,2}$ \\ ${ }^{1}$ Department of Mathematics, Faculty of Science, Naresuan University, Phitsanulok 65000, Thailand \\ 2 PERDO National Centre of Excellence in Mathematics, Faculty of Science, Mahidol University, Bangkok \\ 10400, Thailand
}

Correspondence should be addressed to Somyot Plubtieng, somyotp@nu.ac.th

Received 12 February 2009; Accepted 18 May 2009

Recommended by William A. Kirk

We introduce an iterative method for finding a common element of the set of common fixed points of a countable family of nonexpansive mappings, the set of solutions of a variational inclusion with set-valued maximal monotone mapping, and inverse strongly monotone mappings and the set of solutions of an equilibrium problem in Hilbert spaces. Under suitable conditions, some strong convergence theorems for approximating this common elements are proved. The results presented in the paper improve and extend the main results of J. W. Peng et al. (2008) and many others.

Copyright $(2009$ S. Plubtieng and W. Sriprad. This is an open access article distributed under the Creative Commons Attribution License, which permits unrestricted use, distribution, and reproduction in any medium, provided the original work is properly cited.

\section{Introduction}

Let $H$ be a real Hilbert space whose inner product and norm are denoted by $\langle\cdot, \cdot\rangle$ and $\|\cdot\|$, respectively. Let $C$ be a nonempty closed convex subset of $H$, and let $F$ be a bifunction of $C \times C$ into $\mathbb{R}$, where $\mathbb{R}$ is the set of real numbers. The equilibrium problem for $F: C \times C \rightarrow \mathbb{R}$ is to find $x \in C$ such that

$$
F(x, y) \geq 0, \quad \forall y \in C
$$

The set of solutions of (1.1) is denoted by $E P(F)$. Recently, Combettes and Hirstoaga [1] introduced an iterative scheme of finding the best approximation to the initial data when $E P(F)$ is nonempty and proved a strong convergence theorem. Let $A: C \rightarrow H$ be a nonlinear 
map. The classical variational inequality which is denoted by $V I(A, C)$ is to find $u \in C$ such that

$$
\langle A u, v-u\rangle \geq 0, \quad \forall v \in C
$$

The variational inequality has been extensively studied in literature. See, for example, $[2,3]$ and the references therein. Recall that a mapping $T$ of $C$ into itself is called nonexpansive if

$$
\|S u-S v\| \leq\|u-v\|, \quad \forall u, v \in C
$$

A mapping $f: C \rightarrow C$ is called contractive if there exists a constant $\beta \in(0,1)$ such that

$$
\|f u-f v\| \leq \beta\|u-v\|, \quad \forall u, v \in C
$$

We denote by $F(S)$ the set of fixed points of $S$.

Some methods have been proposed to solve the equilibrium problem and fixed point problem of nonexpansive mapping; see, for instance, [3-6] and the references therein. Recently, Plubtieng and Punpaeng [6] introduced the following iterative scheme. Let $x_{1} \in H$ and let $\left\{x_{n}\right\}$, and $\left\{u_{n}\right\}$ be sequences generated by

$$
\begin{gathered}
F\left(u_{n}, y\right)+\frac{1}{r_{n}}\left\langle y-u_{n}, u_{n}-x_{n}\right\rangle \geq 0, \quad \forall y \in H, \\
x_{n+1}=\alpha_{n} \gamma f\left(x_{n}\right)+\left(I-\alpha_{n} A\right) S u_{n}, \quad \forall n \in \mathbb{N} .
\end{gathered}
$$

They proved that if the sequences $\left\{\alpha_{n}\right\}$ and $\left\{r_{n}\right\}$ of parameters satisfy appropriate conditions, then the sequences $\left\{x_{n}\right\}$ and $\left\{u_{n}\right\}$ both converge strongly to the unique solution of the variational inequality

$$
\langle(A-\gamma f) z, z-x\rangle \geq 0, \quad \forall x \in F(S) \cap E P(F),
$$

which is the optimality condition for the minimization problem

$$
\min _{x \in F(S) \cap E P(F)} \frac{1}{2}\langle A x, x\rangle-h(x)
$$

where $h$ is a potential function for $\gamma f$.

Let $A: H \rightarrow H$ be a single-valued nonlinear mapping, and let $M: H \rightarrow 2^{H}$ be a set-valued mapping. We consider the following variational inclusion, which is to find a point $u \in H$ such that

$$
\theta \in A(u)+M(u)
$$

where $\theta$ is the zero vector in $H$. The set of solutions of problem (1.8) is denoted by $I(A, M)$. If $A=0$, then problem (1.8) becomes the inclusion problem introduced by Rockafellar [7]. 
If $M=\partial \delta_{C}$, where $C$ is a nonempty closed convex subset of $H$ and $\delta_{C}: H \rightarrow[0, \infty]$ is the indicator function of $C$, that is,

$$
\delta_{C}(x)= \begin{cases}0, & x \in C, \\ +\infty, & x \notin C,\end{cases}
$$

then the variational inclusion problem (1.8) is equivalent to variational inequality problem (1.2). It is known that (1.8) provides a convenient framework for the unified study of optimal solutions in many optimization related areas including mathematical programming, complementarity, variational inequalities, optimal control, mathematical economics, equilibria, game theory. Also various types of variational inclusions problems have been extended and generalized (see [8] and the references therein.)

Very recently, Peng et al. [9] introduced the following iterative scheme for finding a common element of the set of solutions to the problem (1.8), the set of solutions of an equilibrium problem, and the set of fixed points of a nonexpansive mapping in Hilbert space. Starting with $x_{1} \in H$, define sequence, $\left\{x_{n}\right\},\left\{y_{n}\right\}$, and $\left\{u_{n}\right\}$ by

$$
\begin{gathered}
F\left(u_{n}, y\right)+\frac{1}{r_{n}}\left\langle y-u_{n}, u_{n}-x_{n}\right\rangle \geq 0, \quad \forall y \in H, \\
x_{n+1}=\alpha_{n} \gamma f\left(x_{n}\right)+\left(1-\alpha_{n}\right) S y_{n}, \\
y_{n}=J_{M, \lambda}\left(u_{n}-\lambda A u_{n}\right), \quad \forall n \geq 0,
\end{gathered}
$$

for all $n \in \mathbb{N}$, where $\lambda \in(0,2 \alpha],\left\{\alpha_{n}\right\} \subset[0,1]$ and $\left\{r_{n}\right\} \subset(0, \infty)$. They proved that under certain appropriate conditions imposed on $\left\{\alpha_{n}\right\}$ and $\left\{r_{n}\right\}$, the sequences $\left\{x_{n}\right\},\left\{y_{n}\right\}$, and $\left\{u_{n}\right\}$ generated by (1.10) converge strongly to $z \in F(T) \cap I(A, M) \cap E P(F)$, where $z=P_{F(S) \cap I(A, M) \cap E P(F)} f(z)$.

Motivated and inspired by Plubtieng and Punpaeng [6], Peng et al. [9] and Aoyama et al. [10], we introduce an iterative scheme for finding a common element of the set of solutions of the variational inclusion problem (1.8) with multi-valued maximal monotone mapping and inverse-strongly monotone mappings, the set of solutions of an equilibrium problem and the set of fixed points of a nonexpansive mapping in Hilbert space. Starting with an arbitrary $x_{1} \in H$, define sequences $\left\{x_{n}\right\},\left\{y_{n}\right\}$ and $\left\{u_{n}\right\}$ by

$$
\begin{gathered}
F\left(u_{n}, y\right)+\frac{1}{r_{n}}\left\langle y-u_{n}, u_{n}-x_{n}\right\rangle \geq 0, \quad \forall y \in H, \\
x_{n+1}=\alpha_{n} \gamma f\left(x_{n}\right)+\left(I-\alpha_{n} B\right) S_{n} y_{n}, \\
y_{n}=J_{M, \lambda}\left(u_{n}-\lambda A u_{n}\right), \quad \forall n \geq 0,
\end{gathered}
$$

for all $n \in \mathbb{N}$, where $\lambda \in(0,2 \alpha],\left\{\alpha_{n}\right\} \subset[0,1]$, and let $\left\{r_{n}\right\} \subset(0, \infty) ; B$ be a strongly bounded linear operator on $H$, and $\left\{S_{n}\right\}$ is a sequence of nonexpansive mappings on $H$. Under suitable conditions, some strong convergence theorems for approximating to this common elements are proved. Our results extend and improve some corresponding results in $[3,9]$ and the references therein. 


\section{Preliminaries}

This section collects some lemmas which will be used in the proofs for the main results in the next section.

Let $H$ be a real Hilbert space with inner product $\langle\cdot, \cdot\rangle$ and norm $\|\cdot\|$, respectively.

It is wellknown that for all $x, y \in H$ and $\lambda \in[0,1]$, there holds

$$
\|\lambda x+(1-\lambda) y\|^{2}=\lambda\|x\|^{2}+(1-\lambda)\|y\|^{2}-\lambda(1-\lambda)\|x-y\|^{2} .
$$

Let $C$ be a nonempty closed convex subset of $H$. Then, for any $x \in H$, there exists a unique nearest point of $C$, denoted by $P_{C} x$, such that $\left\|x-P_{C} x\right\| \leq\|x-y\|$ for all $y \in C$. Such a $P_{C}$ is called the metric projection from $H$ into $C$. We know that $P_{C}$ is nonexpansive. It is also known that, $P_{C} x \in C$ and

$$
\left\langle x-P_{C} x, P_{C} x-z\right\rangle \geq 0, \quad \forall x \in H \text { and } z \in C .
$$

It is easy to see that (2.2) is equivalent to

$$
\|x-z\|^{2} \geq\left\|x-P_{C} x\right\|^{2}+\left\|z-P_{C} x\right\|^{2}, \quad \forall x \in H, z \in C .
$$

For solving the equilibrium problem for a bifunction $F: C \times C \rightarrow \mathbb{R}$, let us assume that $F$ satisfies the following conditions:

(A1) $F(x, x)=0$ for all $x \in C$;

(A2) $F$ is monotone, that is, $F(x, y)+F(y, x) \leq 0$ for all $x, y \in C$;

(A3) for each $x, y, z \in C$,

$$
\lim _{t \rightarrow 0} F(t z+(1-t) x, y) \leq F(x, y)
$$

(A4) for each $x \in C, y \mapsto F(x, y)$ is convex and lower semicontinuous.

The following lemma appears implicitly in [11] and [1].

Lemma 2.1 (See $[1,11])$. Let $C$ be a nonempty closed convex subset of $H$ and let $F$ be a bifunction of $C \times C$ in to $\mathbb{R}$ satisfying (A1)-(A4). Let $r>0$ and $x \in H$. Then, there exists $z \in C$ such that

$$
F(z, y)+\frac{1}{r}\langle y-z, z-x\rangle \geq 0, \quad \forall y \in C
$$

Define a mapping $T_{r}: H \rightarrow C$ as follows:

$$
T_{r}(x)=\left\{z \in C: F(z, y)+\frac{1}{r}\langle y-z, z-x\rangle \geq 0, \forall y \in C\right\},
$$


for all $z \in H$. Then, the following hold:

(1) $T_{r}$ is single-valued;

(2) $T_{r}$ is firmly nonexpansive, that is, for any $x, y \in H$,

$$
\left\|T_{r} x-T_{r} y\right\|^{2} \leq\left\langle T_{r} x-T_{r} y, x-y\right\rangle
$$

(3) $F\left(T_{r}\right)=E P(F)$;

(4) $E P(F)$ is closed and convex.

We also need the following lemmas for proving our main result.

Lemma 2.2 (See [12]). Let $H$ be a Hilbert space, $C$ a nonempty closed convex subset of $H, f: H \rightarrow$ $H$ a contraction with coefficient $0<\alpha<1$, and $B$ a strongly positive linear bounded operator with coefficient $\bar{\gamma}>0$. Then :

(1) if $0<\gamma<\bar{\gamma} / \alpha$, then $\langle x-y,(B-\gamma f) x-(B-\gamma f) y\rangle \geq(\bar{\gamma}-\gamma \alpha)\|x-y\|^{2}, x, y \in H$.

(2) if $0<\rho<\|B\|^{-1}$, then $\|I-\rho B\| \leq 1-\rho \bar{\gamma}$.

Lemma 2.3 (See [13]). Assume $\left\{a_{n}\right\}$ is a sequence of nonnegative real numbers such that

$$
a_{n+1} \leq\left(1-\gamma_{n}\right) a_{n}+\delta_{n}, \quad n \geq 0
$$

where $\left\{\gamma_{n}\right\}$ is a sequence in $(0,1)$ and $\left\{\delta_{n}\right\}$ is a sequence in $\mathbb{R}$ such that

(1) $\sum_{n=1}^{\infty} \gamma_{n}=\infty$;

(2) $\lim \sup _{n \rightarrow \infty} \delta_{n} / \gamma_{n} \leq 0$ or $\sum_{n=1}^{\infty}\left|\delta_{n}\right|<\infty$.

Then $\lim _{n \rightarrow \infty} a_{n}=0$.

Recall that a mapping $A: H \rightarrow H$ is called $\alpha$-inverse-strongly monotone, if there exists a positive number $\alpha$ such that

$$
\langle A u-A v, u-v\rangle \geq \alpha\|A u-A v\|^{2}, \quad \forall u, v \in H .
$$

Let $I$ be the identity mapping on $H$. It is well known that if $A: H \rightarrow H$ is $\alpha$-inversestrongly monotone, then $A$ is $1 / \alpha$-Lipschitz continuous and monotone mapping. In addition, if $0<\lambda \leq 2 \alpha$, then $I-\lambda A$ is a nonexpansive mapping.

A set-valued $M: H \rightarrow 2^{H}$ is called monotone if for all $x, y \in H, f \in M x$ and $g \in$ $M y$ imply $\langle x-y, f-g\rangle \geq 0$. A monotone mapping $M: H \rightarrow 2^{H}$ is maximal if its graph $G(M):=\{(x, f) \in H \times H \mid f \in M(x)\}$ of $M$ is not properly contained in the graph of any other monotone mapping. It is known that a monotone mapping $M$ is maximal if and only if for $(x, f) \in H \times H,\langle x-y, f-g\rangle \geq 0$ for every $(y, g) \in G(M)$ implies $f \in M x$.

Let the set-valued mapping $M: H \rightarrow 2^{H}$ be maximal monotone. We define the resolvent operator $J_{M, \lambda}$ associated with $M$ and $\lambda$ as follows:

$$
J_{M, \lambda}(u)=(I+\lambda M)^{-1}(u), \quad \forall u \in H
$$


where $\lambda$ is a positive number. It is worth mentioning that the resolvent operator $J_{M, \lambda}$ is single-valued, nonexpansive and 1-inverse-strongly monotone, see for example [14] and that a solution of problem (1.8) is a fixed point of the operator $J_{M, \lambda}(I-\lambda A)$ for all $\lambda>0$, see for instance [15].

Lemma 2.4 (See [14]). Let $M: H \rightarrow 2^{H}$ be a maximal monotone mapping and $A: H \rightarrow H$ be a Lipschitz-continuous mapping. Then the mapping $S=M+A: H \rightarrow 2^{H}$ is a maximal monotone mapping.

Remark 2.5 (See [9]). Lemma 2.4 implies that $I(A, M)$ is closed and convex if $M: H \rightarrow 2^{H}$ is a maximal monotone mapping and $A: H \rightarrow H$ be an inverse strongly monotone mapping.

Lemma 2.6 (See [10]). Let $C$ be a nonempty closed subset of a Banach space and let $\left\{S_{n}\right\}$ a sequence of mappings of $C$ into itself. Suppose that $\sum_{n=1}^{\infty} \sup \left\{\left\|S_{n+1} z-S_{n} z\right\|: z \in C\right\}<\infty$. Then, for each $x \in C,\left\{S_{n} x\right\}$ converges strongly to some point of $C$. Moreover, let $S$ be a mapping from $C$ into itself defined by

$$
S x=\lim _{n \rightarrow \infty} S_{n} x, \quad \forall x \in C .
$$

Then $\lim _{n \rightarrow \infty} \sup \left\{\left\|S z-S_{n} z\right\|: z \in C\right\}=0$.

\section{Main Results}

We begin this section by proving a strong convergence theorem of an implicit iterative sequence $\left\{x_{n}\right\}$ obtained by the viscosity approximation method for finding a common element of the set of solutions of the variational inclusion, the set of solutions of an equilibrium problem and the set of fixed points of a nonexpansive mapping.

Throughout the rest of this paper, we always assume that $f$ is a contraction of $H$ into itself with coefficient $\beta \in(0,1)$, and $B$ is a strongly positive bounded linear operator with coefficient $\bar{\gamma}$ and $0<\gamma<\bar{\gamma} / \beta$. Let $S$ be a nonexpansive mapping of $H$ into $H$. Let $A: H \rightarrow H$ be an $\alpha$-inverse-strongly monotone mapping, $M: H \rightarrow 2^{H}$ be a maximal monotone mapping and let $J_{M, \lambda}$ be defined as in (2.10). Let $\left\{T_{r_{n}}\right\}$ be a sequence of mappings defined as Lemma 2.1. Consider a sequence of mappings $\left\{S_{n}\right\}$ on $H$ defined by

$$
S_{n} x=\alpha_{n} \gamma f(x)+\left(I-\alpha_{n} B\right) S J_{M, \lambda}(I-\lambda A) T_{r_{n}} x, \quad x \in H, n \geq 1,
$$

where $\left\{\alpha_{n}\right\} \subset\left(0,\|B\|^{-1}\right)$. By Lemma 2.2, we note that $S_{n}$ is a contraction. Therefore, by the Banach contraction principle, $S_{n}$ has a unique fixed point $x_{n} \in H$ such that

$$
x_{n}=\alpha_{n} \gamma f\left(x_{n}\right)+\left(I-\alpha_{n} B\right) S J_{M, \lambda}(I-\lambda A) T_{r_{n}} x_{n} .
$$

Theorem 3.1. Let $H$ be a real Hilbert space, let $F$ be a bifunction from $H \times H \rightarrow \mathbb{R}$ satisfying (A1)-(A4) and let $S$ be a nonexpansive mapping on $H$. Let $A: H \rightarrow H$ be an $\alpha$-inverse-strongly monotone mapping, $M: H \rightarrow 2^{H}$ be a maximal monotone mapping such that $\Omega:=F(S) \cap E P(F) \cap$ $I(A, M) \neq \varnothing$. Let $f$ be a contraction of $H$ into itself with a constant $\beta \in(0,1)$ and let $B$ be a strongly 
bounded linear operator on $H$ with coefficient $\bar{\gamma}>0$ and $0<\gamma<\bar{\gamma} / \beta$. Let $\left\{x_{n}\right\},\left\{y_{n}\right\}$ and $\left\{u_{n}\right\}$ be sequences generated by $x_{1} \in H$ and

$$
\begin{gathered}
F\left(u_{n}, y\right)+\frac{1}{r_{n}}\left\langle y-u_{n}, u_{n}-x_{n}\right\rangle \geq 0, \quad \forall y \in H \\
x_{n}=\alpha_{n} \gamma f\left(x_{n}\right)+\left(I-\alpha_{n} B\right) S y_{n} \\
y_{n}=J_{M, \lambda}\left(u_{n}-\lambda A u_{n}\right) \quad \forall n \geq 0
\end{gathered}
$$

where $\lambda \in(0,2 \alpha],\left\{r_{n}\right\} \subset(0, \infty)$ and $\left\{\alpha_{n}\right\} \subset[0,1]$ satisfy $\lim _{n \rightarrow \infty} \alpha_{n}=0$ and $\liminf _{n \rightarrow \infty} r_{n}>$ 0 . Then, $\left\{x_{n}\right\},\left\{y_{n}\right\}$ and $\left\{u_{n}\right\}$ converges strongly to a point $z$ in $\Omega$ which solves the variational inequality:

$$
\langle(B-\gamma f) z, z-x\rangle \leq 0, \quad x \in \Omega
$$

Equivalently, we have $z=P_{\Omega}(I-B+\gamma f)(z)$.

Proof. First, we assume that $\alpha_{n} \in\left(0,\|B\|^{-1}\right)$. By Lemma 2.2, we obtain $\left\|I-\alpha_{n} B\right\| \leq 1-\alpha_{n} \bar{\gamma}$. Let $v \in \Omega$. Since $u_{n}=T_{r_{n}} x_{n}$, we have

$$
\left\|u_{n}-v\right\|=\left\|T_{r_{n}} x_{n}-T_{r_{n}} v\right\| \leq\left\|x_{n}-v\right\| \quad \forall n \in \mathbb{N}
$$

We note from $v \in \Omega$ that $v=J_{M, \lambda}(v-\lambda A v)$. As $I-\lambda A$ is nonexpansive, we have

$$
\begin{aligned}
\left\|y_{n}-v\right\| & =\left\|J_{M, \lambda}\left(u_{n}-\lambda A u_{n}\right)-J_{M, \lambda}(v-\lambda A v)\right\| \\
& \leq\left\|\left(u_{n}-\lambda A u_{n}\right)-(v-\lambda A v)\right\| \leq\left\|u_{n}-v\right\| \leq\left\|x_{n}-v\right\|
\end{aligned}
$$

for all $n \in \mathbb{N}$. Thus, we have

$$
\begin{aligned}
\left\|x_{n}-v\right\| & =\left\|\alpha_{n} \gamma f\left(x_{n}\right)+\left(I-\alpha_{n} B\right) S y_{n}-v\right\| \\
& \leq \alpha_{n}\left\|\gamma f\left(x_{n}\right)-B v\right\|+\left\|I-\alpha_{n} B\right\|\left\|y_{n}-v\right\| \\
& \leq \alpha_{n}\left\|\gamma f\left(x_{n}\right)-B v\right\|+\left(1-\alpha_{n} \bar{\gamma}\right)\left\|x_{n}-v\right\| \\
& \leq \alpha_{n}\left\|\gamma\left(f\left(x_{n}\right)-f(v)\right)+(\gamma f(v)-B v)\right\|+\left(1-\alpha_{n} \bar{\gamma}\right)\left\|x_{n}-v\right\| \\
& \leq \alpha_{n} \gamma \beta\left\|x_{n}-v\right\|+\alpha_{n}\|\gamma f(v)-B v\|+\left(1-\alpha_{n} \bar{\gamma}\right)\left\|x_{n}-v\right\| \\
& =\left(1-\alpha_{n}(\bar{\gamma}-\gamma \beta)\right)\left\|x_{n}-v\right\|+\alpha_{n}\|\gamma f(v)-B v\| .
\end{aligned}
$$

It follows that $\left\|x_{n}-v\right\| \leq\|\gamma f(v)-B v\| /(\bar{\gamma}-\gamma \beta), \forall n \geq 1$. Hence $\left\{x_{n}\right\}$ is bounded and we also obtain that $\left\{u_{n}\right\},\left\{y_{n}\right\},\left\{f\left(x_{n}\right)\right\},\left\{S y_{n}\right\}$ and $\left\{A u_{n}\right\}$ are bounded. Next, we show that $\| y_{n}-$ $S y_{n} \| \rightarrow 0$. Since $\alpha_{n} \rightarrow 0$, we note that

$$
\left\|x_{n}-S y_{n}\right\|=\alpha_{n}\left\|\gamma f\left(x_{n}\right)-B S y_{n}\right\| \longrightarrow 0 \quad \text { as } n \longrightarrow \infty
$$


Moreover, it follows from Lemma 2.1 that

$$
\begin{aligned}
\left\|u_{n}-v\right\|^{2} & =\left\|T_{r_{n}} x_{n}-T_{r_{n}} v\right\|^{2} \leq\left\langle T_{r_{n}} x_{n}-T_{r_{n}} v, x_{n}-v\right\rangle=\left\langle u_{n}-v, x_{n}-v\right\rangle \\
& =\frac{1}{2}\left(\left\|u_{n}-v\right\|^{2}+\left\|x_{n}-v\right\|^{2}-\left\|x_{n}-u_{n}\right\|^{2}\right),
\end{aligned}
$$

and hence $\left\|u_{n}-v\right\|^{2} \leq\left\|x_{n}-v\right\|^{2}-\left\|x_{n}-u_{n}\right\|^{2}$. Therefore, we have

$$
\begin{aligned}
\left\|x_{n}-v\right\|^{2}= & \left\|\alpha_{n} \gamma f\left(x_{n}\right)+\left(I-\alpha_{n} B\right) S y_{n}-v\right\|^{2} \\
= & \left\|\left(I-\alpha_{n} B\right)\left(S y_{n}-v\right)+\alpha_{n}\left(\gamma f\left(x_{n}\right)-B v\right)\right\|^{2} \\
\leq & \left(1-\alpha_{n} \bar{\gamma}\right)^{2}\left\|S y_{n}-v\right\|^{2}+2 \alpha_{n}\left\langle\gamma f\left(x_{n}\right)-B v, x_{n}-v\right\rangle \\
\leq & \left(1-\alpha_{n} \bar{\gamma}\right)^{2}\left\|y_{n}-v\right\|^{2}+2 \alpha_{n} \gamma\left\langle f\left(x_{n}\right)-f(v), x_{n}-v\right\rangle+2 \alpha_{n}\left\langle\gamma f(v)-B v, x_{n}-v\right\rangle \\
\leq & \left(1-\alpha_{n} \bar{\gamma}\right)^{2}\left\|u_{n}-v\right\|^{2}+2 \alpha_{n} \gamma\left\langle f\left(x_{n}\right)-f(v), x_{n}-v\right\rangle+2 \alpha_{n}\left\langle\gamma f(v)-B v, x_{n}-v\right\rangle \\
\leq & \left(1-\alpha_{n} \bar{\gamma}\right)^{2}\left\{\left\|x_{n}-v\right\|^{2}-\left\|x_{n}-u_{n}\right\|^{2}\right\}+2 \alpha_{n} \gamma \beta\left\|x_{n}-v\right\|^{2} \\
& +2 \alpha_{n}\|\gamma f(v)-B v\|\left\|x_{n}-v\right\| \\
= & \left(1-2 \alpha_{n}(\bar{\gamma}-\gamma \beta)+\left(\alpha_{n} \bar{\gamma}\right)^{2}\right)\left\|x_{n}-v\right\|^{2}-\left(1-\alpha_{n} \bar{\gamma}\right)^{2}\left\|x_{n}-u_{n}\right\|^{2} \\
& +2 \alpha_{n}\|\gamma f(v)-B v\|\left\|x_{n}-v\right\| \\
\leq & \left\|x_{n}-v\right\|^{2}+\alpha_{n} \bar{\gamma}^{2}\left\|x_{n}-v\right\|^{2}-\left(1-\alpha_{n} \bar{\gamma}\right)^{2}\left\|x_{n}-u_{n}\right\|^{2}+2 \alpha_{n}\|\gamma f(v)-B v\|\left\|x_{n}-v\right\|,
\end{aligned}
$$

and hence

$$
\left(1-\alpha_{n} \bar{\gamma}\right)^{2}\left\|x_{n}-u_{n}\right\|^{2} \leq \alpha_{n} \bar{\gamma}^{2}\left\|x_{n}-v\right\|^{2}+2 \alpha_{n}\|\gamma f(v)-B v\|\left\|x_{n}-v\right\|
$$

Since $\left\{x_{n}\right\}$ is bounded and $\alpha_{n} \rightarrow 0$, it follows that $\left\|x_{n}-u_{n}\right\| \rightarrow 0$ as $n \rightarrow \infty$.

Put $M=\sup _{n \geq 1}\left\{\|\gamma f(v)-B v\|\left\|x_{n}-v\right\|\right\}$. From (3.10), it follows by the nonexpansive of $J_{M, \lambda}$ and the inverse strongly monotonicity of $A$ that

$$
\begin{aligned}
\left\|x_{n}-v\right\|^{2} & \leq\left(1-\alpha_{n} \bar{\gamma}\right)^{2}\left\|y_{n}-v\right\|^{2}+2 \alpha_{n} \gamma \beta\left\|x_{n}-v\right\|^{2}+2 \alpha_{n} M \\
& \leq\left(1-\alpha_{n} \bar{\gamma}\right)^{2}\left\|\left(u_{n}-\lambda A u_{n}\right)-(v-\lambda A v)\right\|^{2}+2 \alpha_{n} \gamma \beta\left\|x_{n}-v\right\|^{2}+2 \alpha_{n} M \\
& \leq\left(1-\alpha_{n} \bar{\gamma}\right)^{2}\left\{\left\|u_{n}-v\right\|^{2}+\lambda(\lambda-2 \alpha)\left\|A u_{n}-A v\right\|^{2}\right\}+2 \alpha_{n} \gamma \beta\left\|x_{n}-v\right\|^{2}+2 \alpha_{n} M \\
& \leq\left(1-\alpha_{n} \bar{\gamma}\right)^{2}\left\|x_{n}-v\right\|^{2}+\left(1-\alpha_{n} \bar{\gamma}\right)^{2} \lambda(\lambda-2 \alpha)\left\|A u_{n}-A v\right\|^{2}+2 \alpha_{n} \gamma \beta\left\|x_{n}-v\right\|^{2}+2 \alpha_{n} M \\
& =\left(1-2 \alpha_{n}(\bar{\gamma}-\gamma \beta)+\left(\alpha_{n} \bar{\gamma}\right)^{2}\right)\left\|x_{n}-v\right\|^{2}+\left(1-\alpha_{n} \bar{\gamma}\right)^{2} \lambda(\lambda-2 \alpha)\left\|A u_{n}-A v\right\|^{2}+2 \alpha_{n} M \\
& \leq\left\|x_{n}-v\right\|^{2}+\alpha_{n} \bar{\gamma}^{2}\left\|x_{n}-v\right\|^{2}+\left(1-\alpha_{n} \bar{\gamma}\right)^{2} \lambda(\lambda-2 \alpha)\left\|A u_{n}-A v\right\|^{2}+2 \alpha_{n} M,
\end{aligned}
$$


Fixed Point Theory and Applications

which implies that

$$
\left(1-\alpha_{n} \bar{\gamma}\right)^{2} \lambda(2 \alpha-\lambda)\left\|A u_{n}-A v\right\|^{2} \leq \alpha_{n} \bar{\gamma}^{2}\left\|x_{n}-v\right\|^{2}+2 \alpha_{n} M
$$

Since $\alpha_{n} \rightarrow 0$, we have $\left\|A u_{n}-A v\right\| \rightarrow 0$ as $n \rightarrow \infty$. Since $J_{M, \lambda}$ is 1-inverse-strongly monotone and $I-\lambda A$ is nonexpansive, we have

$$
\begin{aligned}
\left\|y_{n}-v\right\|^{2} & =\left\|J_{M, \lambda}\left(u_{n}-\lambda A u_{n}\right)-J_{M, \lambda}(v-\lambda A v)\right\|^{2} \leq\left\langle u_{n}-\lambda A u_{n}-(v-\lambda A v), y_{n}-v\right\rangle \\
& =\frac{1}{2}\left\{\left\|u_{n}-\lambda A u_{n}-(v-\lambda A v)\right\|^{2}+\left\|y_{n}-v\right\|^{2}-\left\|u_{n}-\lambda A u_{n}-(v-\lambda A v)-\left(y_{n}-v\right)\right\|^{2}\right\} \\
& \leq \frac{1}{2}\left\{\left\|u_{n}-v\right\|^{2}+\left\|y_{n}-v\right\|^{2}-\left\|u_{n}-y_{n}-\lambda\left(A u_{n}-A v\right)\right\|^{2}\right\} \\
& =\frac{1}{2}\left\{\left\|u_{n}-v\right\|^{2}+\left\|y_{n}-v\right\|^{2}-\left\|u_{n}-y_{n}\right\|^{2}+2 \lambda\left\langle u_{n}-y_{n}, A u_{n}-A v\right\rangle-\lambda^{2}\left\|A u_{n}-A v\right\|^{2}\right\} .
\end{aligned}
$$

Thus, we have

$$
\left\|y_{n}-v\right\|^{2} \leq\left\|u_{n}-v\right\|^{2}-\left\|u_{n}-y_{n}\right\|^{2}+2 \lambda\left\langle u_{n}-y_{n}, A u_{n}-A v\right\rangle-\lambda^{2}\left\|A u_{n}-A v\right\|^{2} .
$$

From (3.5), (3.10), and (3.15), we have

$$
\begin{aligned}
\left\|x_{n}-v\right\|^{2} \leq & \left(1-\alpha_{n} \bar{\gamma}\right)^{2}\left\|y_{n}-v\right\|^{2}+2 \alpha_{n} \gamma \beta\left\|x_{n}-v\right\|^{2}+2 \alpha_{n} M \\
\leq & \left(1-\alpha_{n} \bar{\gamma}\right)^{2}\left\{\left\|u_{n}-v\right\|^{2}-\left\|u_{n}-y_{n}\right\|^{2}+2 \lambda\left\langle u_{n}-y_{n}, A u_{n}-A v\right\rangle-\lambda^{2}\left\|A u_{n}-A v\right\|^{2}\right\} \\
& +2 \alpha_{n} \gamma \beta\left\|x_{n}-v\right\|^{2}+2 \alpha_{n} M \\
\leq & \left(1-\alpha_{n} \bar{\gamma}\right)^{2}\left\|x_{n}-v\right\|^{2}-\left(1-\alpha_{n} \bar{\gamma}\right)^{2}\left\|u_{n}-y_{n}\right\|^{2}+2\left(1-\alpha_{n} \bar{\gamma}\right)^{2} \lambda\left\langle u_{n}-y_{n}, A u_{n}-A v\right\rangle \\
& -\left(1-\alpha_{n} \bar{\gamma}\right)^{2} \lambda^{2}\left\|A u_{n}-A v\right\|^{2}+2 \alpha_{n} \gamma \beta\left\|x_{n}-v\right\|^{2}+2 \alpha_{n} M \\
= & \left(1-2 \alpha_{n}(\bar{\gamma}-\gamma \beta)+\left(\alpha_{n} \bar{\gamma}\right)^{2}\right)\left\|x_{n}-v\right\|^{2}-\left(1-\alpha_{n} \bar{\gamma}\right)^{2}\left\|u_{n}-y_{n}\right\|^{2} \\
& +2\left(1-\alpha_{n} \bar{\gamma}\right)^{2} \lambda\left\langle u_{n}-y_{n}, A u_{n}-A v\right\rangle-\left(1-\alpha_{n} \bar{\gamma}\right)^{2} \lambda^{2}\left\|A u_{n}-A v\right\|^{2}+2 \alpha_{n} M \\
\leq & \left\|x_{n}-v\right\|^{2}+\alpha_{n} \bar{\gamma}^{2}\left\|x_{n}-v\right\|^{2}-\left(1-\alpha_{n} \bar{\gamma}\right)^{2}\left\|u_{n}-y_{n}\right\|^{2} \\
& +2\left(1-\alpha_{n} \bar{\gamma}\right)^{2} \lambda\left\langle u_{n}-y_{n}, A u_{n}-A v\right\rangle-\left(1-\alpha_{n} \bar{\gamma}\right)^{2} \lambda^{2}\left\|A u_{n}-A v\right\|^{2}+2 \alpha_{n} M .
\end{aligned}
$$

Thus, we get

$$
\begin{gathered}
\left(1-\alpha_{n} \bar{\gamma}\right)^{2}\left\|u_{n}-y_{n}\right\|^{2} \leq \\
\alpha_{n} \bar{\gamma}^{2}\left\|x_{n}-v\right\|^{2}+2\left(1-\alpha_{n} \bar{\gamma}\right)^{2} \lambda\left\langle u_{n}-y_{n}, A u_{n}-A v\right\rangle \\
-\left(1-\alpha_{n} \bar{\gamma}\right)^{2} \lambda^{2}\left\|A u_{n}-A v\right\|^{2}+2 \alpha_{n} M .
\end{gathered}
$$


Since $\alpha_{n} \rightarrow 0,\left\|A u_{n}-A v\right\| \rightarrow 0$ as $n \rightarrow \infty$, we have $\left\|u_{n}-y_{n}\right\| \rightarrow 0$ as $n \rightarrow \infty$. It follows from the inequality $\left\|y_{n}-S y_{n}\right\| \leq\left\|y_{n}-u_{n}\right\|+\left\|u_{n}-x_{n}\right\|+\left\|x_{n}-S y_{n}\right\|$ that $\left\|y_{n}-S y_{n}\right\| \rightarrow 0$ as $n \rightarrow \infty$. Moreover, we have $\left\|x_{n}-y_{n}\right\| \leq\left\|x_{n}-u_{n}\right\|+\left\|u_{n}-y_{n}\right\| \rightarrow 0$ as $n \rightarrow \infty$.

Put $U \equiv S J_{M, \lambda}(I-\lambda A)$. Since both $S$ and $J_{M, \lambda}(I-\lambda A)$ are nonexpansive, we have $U$ is a nonexpansive mapping on $H$ and then we have $x_{n}=\alpha_{n} \gamma f\left(x_{n}\right)+\left(I-\alpha_{n} B\right) U T_{r_{n}} x_{n}$ for all $n \in \mathbb{N}$. It follows by Theorem 3.1 of Plubtieng and Punpaeng [6] that $\left\{x_{n}\right\}$ converges strongly to $z \in F(U) \cap E P(F)$, where $z=P_{F(U) \cap E P(F)}(\gamma f+(I-B)) z$ and $\langle(B-\gamma f) z, u-z\rangle \geq 0$, for all $u \in F(U) \cap E P(F)$. We will show that $z \in F(S) \cap I(A, M)$. Since $\left\{x_{n}\right\}$ converges strongly to $z$, we also have $x_{n}-z$. Let us show $z \in F(S)$. Assume $z \notin F(S)$. Since $\left\|x_{n}-y_{n}\right\| \rightarrow 0$ and $x_{n} \rightarrow z$, we have $y_{n} \rightarrow z$ Since $z \neq S z$, it follows by the Opial's condition that

$$
\begin{aligned}
\liminf _{n \rightarrow \infty}\left\|y_{n}-z\right\| & <\liminf _{n \rightarrow \infty}\left\|y_{n}-S z\right\| \leq \liminf _{n \rightarrow \infty}\left\{\left\|y_{n}-S y_{n}\right\|+\left\|S y_{n}-S z\right\|\right\} \\
& \leq \liminf _{n \rightarrow \infty}\left\|y_{n}-z\right\| .
\end{aligned}
$$

This is a contradiction. Hence $z \in F(S)$. We now show that $z \in I(A, M)$. In fact, since $A$ is $\alpha$-inverse-strongly monotone, $A$ is an $1 / \alpha$-Lipschitz continuous monotone mapping and $D(A)=H$. It follows from Lemma 2.4 that $M+A$ is maximal monotone. Let $(p, g) \in G(M+A)$, that is, $g-A p \in M(p)$. Again since $y_{n}=J_{M, \lambda}\left(u_{n}-\lambda A u_{n}\right)$, we have $u_{n}-\lambda A u_{n} \in(I+\lambda M)\left(y_{n}\right)$, that is,

$$
\frac{1}{\lambda}\left(u_{n}-y_{n}-\lambda A u_{n}\right) \in M\left(y_{n}\right)
$$

By the maximal monotonicity of $M+A$, we have

$$
\left\langle p-y_{n}, g-A p-\frac{1}{\lambda}\left(u_{n}-y_{n}-\lambda A u_{n}\right)\right\rangle \geq 0,
$$

and so

$$
\begin{aligned}
\left\langle p-y_{n}, g\right\rangle & \geq\left\langle p-y_{n}, A p+\frac{1}{\lambda}\left(u_{n}-y_{n}-\lambda A u_{n}\right)\right\rangle \\
& =\left\langle p-y_{n}, A p-A y_{n}+A y_{n}-A u_{n}+\frac{1}{\lambda}\left(u_{n}-y_{n}\right)\right\rangle \\
& \geq 0+\left\langle p-y_{n}, A y_{n}-A u_{n}\right\rangle+\left\langle p-y_{n}, \frac{1}{\lambda}\left(u_{n}-y_{n}\right)\right\rangle
\end{aligned}
$$

It follows from $\left\|u_{n}-y_{n}\right\| \rightarrow 0,\left\|A u_{n}-A y_{n}\right\| \rightarrow 0$ and $y_{n} \rightarrow z$ that

$$
\lim _{n \rightarrow \infty}\left\langle p-y_{n}, g\right\rangle=\langle p-z, g\rangle \geq 0
$$

Since $A+M$ is maximal monotone, this implies that $\theta \in(M+A)(z)$, that is, $z \in I(A, M)$. Hence, $z \in \Omega:=F(S) \cap E P(F) \cap I(A, M)$. Since $F(S) \cap I(A, M)=F(S) \cap F\left(J_{M, \lambda}(I-\lambda A)\right) \subset F(U)$, we have $\Omega \subset F(U) \cap E P(F)$. It implies that $z$ is the unique solution of the variational inequality (3.4). 
Now we prove the following theorem which is the main result of this paper.

Theorem 3.2. Let $H$ be a real Hilbert space, let $F$ be a bifunction from $H \times H \rightarrow \mathbb{R}$ satisfying (A1)-(A4) and let $\left\{S_{n}\right\}$ be a sequence of nonexpansive mappings on $H$. Let $A: H \rightarrow H$ be an $\alpha$-inverse-strongly monotone mapping, $M: H \rightarrow 2^{H}$ be a maximal monotone mapping such that $\Omega:=\bigcap_{n=1}^{\infty} F\left(S_{n}\right) \cap E P(F) \cap I(A, M) \neq \varnothing$. Let $f$ be a contraction of $H$ into itself with a constant $\beta \in(0,1)$ and let $B$ be a strongly bounded linear operator on $H$ with coefficient $\bar{\gamma}>0$ and $0<\gamma<\bar{\gamma} / \beta$. Let $\left\{x_{n}\right\},\left\{y_{n}\right\}$ and $\left\{u_{n}\right\}$ be sequences generated by $x_{1} \in H$ and

$$
\begin{gathered}
F\left(u_{n}, y\right)+\frac{1}{r_{n}}\left\langle y-u_{n}, u_{n}-x_{n}\right\rangle \geq 0, \quad \forall y \in H \\
x_{n+1}=\alpha_{n} \gamma f\left(x_{n}\right)+\left(I-\alpha_{n} B\right) S_{n} y_{n} \\
y_{n}=J_{M, \lambda}\left(u_{n}-\lambda A u_{n}\right) \quad \forall n \geq 0
\end{gathered}
$$

for all $n \in \mathbb{N}$, where $\lambda \in(0,2 \alpha],\left\{\alpha_{n}\right\} \subset[0,1]$ and $\left\{r_{n}\right\} \subset(0, \infty)$ satisfy

$$
\begin{gathered}
\lim _{n \rightarrow \infty} \alpha_{n}=0, \quad \sum_{n=1}^{\infty} \alpha_{n}=\infty, \quad \sum_{n=1}^{\infty}\left|\alpha_{n+1}-\alpha_{n}\right|<\infty, \\
\liminf _{n \rightarrow \infty} r_{n}>0, \quad \sum_{n=1}^{\infty}\left|r_{n+1}-r_{n}\right|<\infty .
\end{gathered}
$$

Suppose that $\sum_{n=1}^{\infty} \sup \left\{\left\|S_{n+1} z-S_{n} z\right\|: z \in K\right\}<\infty$ for any bounded subset $K$ of $H$. Let $S$ be a mapping of $H$ into itself defined by $S x=\lim _{n \rightarrow \infty} S_{n} x$, for all $x \in H$ and suppose that $F(S)=$ $\bigcap_{n=1}^{\infty} F\left(S_{n}\right)$. Then, $\left\{x_{n}\right\},\left\{y_{n}\right\}$ and $\left\{u_{n}\right\}$ converges strongly to $z$, where $z=P_{\Omega}(I-B+\gamma f)(z)$ is a unique solution of the variational inequalities (3.4).

Proof. Since $\alpha_{n} \rightarrow 0$, we may assume that $\alpha_{n} \leq\|B\|^{-1}$ for all $n$. First we will prove that $\left\{x_{n}\right\}$ is bonded. Let $v \in \Omega$. Then, we have

$$
\begin{aligned}
\left\|x_{n+1}-v\right\| & =\left\|\alpha_{n} \gamma f\left(x_{n}\right)+\left(I-\alpha_{n} B\right) S_{n} y_{n}-v\right\| \\
& \leq \alpha_{n}\left\|\gamma f\left(x_{n}\right)-B v\right\|+\left\|I-\alpha_{n} B\right\|\left\|y_{n}-v\right\| \\
& \leq \alpha_{n}\left\|\gamma f\left(x_{n}\right)-B v\right\|+\left(1-\alpha_{n} \bar{\gamma}\right)\left\|x_{n}-v\right\| \\
& \leq \alpha_{n}\left\|\gamma\left(f\left(x_{n}\right)-f(v)\right)+(\gamma f(v)-B v)\right\|+\left(1-\alpha_{n} \bar{\gamma}\right)\left\|x_{n}-v\right\| \\
& \leq \alpha_{n} \gamma \beta\left\|x_{n}-v\right\|+\alpha_{n}\|\gamma f(v)-B v\|+\left(1-\alpha_{n} \bar{\gamma}\right)\left\|x_{n}-v\right\| \\
& =\left(1-\alpha_{n}(\bar{\gamma}-\gamma \beta)\right)\left\|x_{n}-v\right\|+\alpha_{n}\|\gamma f(v)-B v\| \\
& =\left(1-\alpha_{n}(\bar{\gamma}-\gamma \alpha)\right)\left\|x_{n}-v\right\|+\alpha_{n}(\bar{\gamma}-\gamma \alpha) \frac{\|\gamma f(v)-B v\|}{\bar{\gamma}-\gamma \alpha} .
\end{aligned}
$$

It follows from (3.25) and induction that

$$
\left\|x_{n}-v\right\| \leq \max \left\{\left\|x_{1}-v\right\|, \frac{1}{\bar{\gamma}-\gamma \alpha}\|\gamma f(p)-B(p)\|\right\}, \quad n \geq 0
$$


Hence $\left\{x_{n}\right\}$ is bounded and therefore $\left\{u_{n}\right\},\left\{y_{n}\right\},\left\{f\left(x_{n}\right)\right\},\left\{S_{n} y_{n}\right\}$ and $\left\{A\left(u_{n}\right)\right\}$ are also bounded. Next, we show that $\left\|x_{n+1}-x_{n}\right\| \rightarrow 0$. Since $I-\lambda A$ is nonexpansive, it follows that

$$
\begin{aligned}
\left\|y_{n+1}-y_{n}\right\| & =\left\|J_{M, \lambda}\left(u_{n+1}-\lambda A u_{n+1}\right)-J_{M, \lambda}\left(u_{n}-\lambda A u_{n}\right)\right\| \\
& \leq\left\|\left(u_{n+1}-\lambda A u_{n+1}\right)-\left(u_{n}-\lambda A u_{n}\right)\right\| \leq\left\|u_{n+1}-u_{n}\right\| .
\end{aligned}
$$

Then, we have

$$
\begin{aligned}
\left\|x_{n+2}-x_{n+1}\right\|= & \left\|\alpha_{n+1} \gamma f\left(x_{n+1}\right)+\left(I-\alpha_{n+1} B\right) S_{n+1} y_{n+1}-\alpha_{n} \gamma f\left(x_{n}\right)-\left(I-\alpha_{n} B\right) S_{n} y_{n}\right\| \\
= & \| \alpha_{n+1} \gamma f\left(x_{n+1}\right)+\left(I-\alpha_{n+1} B\right) S_{n+1} y_{n+1}-\alpha_{n} \gamma f\left(x_{n}\right)-\left(I-\alpha_{n} B\right) S_{n} y_{n} \\
& -\left(I-\alpha_{n+1} B\right) S_{n+1} y_{n}+\left(I-\alpha_{n+1} B\right) S_{n+1} y_{n}-\left(I-\alpha_{n} B\right) S_{n+1} y_{n} \\
& +\left(I-\alpha_{n} B\right) S_{n+1} y_{n} \| \\
= & \|\left(I-\alpha_{n+1} B\right)\left(S_{n+1} y_{n+1}-S_{n+1} y_{n}\right)+\left(\alpha_{n}-\alpha_{n+1}\right) B S_{n+1} y_{n} \\
& +\left(I-\alpha_{n} B\right)\left(S_{n+1} y_{n}-S_{n} y_{n}\right)+\left(\alpha_{n+1}-\alpha_{n}\right) \gamma f\left(x_{n}\right)+\alpha_{n+1} \gamma f\left(x_{n+1}-f\left(x_{n}\right)\right) \| \\
\leq & \left(1-\alpha_{n+1} \bar{\gamma}\right)\left\|y_{n+1}-y_{n}\right\|+\left|\alpha_{n}-\alpha_{n+1}\right|\left\|B S_{n+1} y_{n}\right\|+\left(1-\alpha_{n} \bar{\gamma}\right)\left\|S_{n+1} y_{n}-S_{n} y_{n}\right\| \\
& +\left|\alpha_{n}-\alpha_{n+1}\right|\left\|\gamma f\left(x_{n}\right)\right\|+\alpha_{n+1} \gamma \beta\left\|x_{n+1}-x_{n}\right\| \\
\leq & \left(1-\alpha_{n+1} \bar{\gamma}\right)\left\|y_{n+1}-y_{n}\right\|+\alpha_{n+1} \gamma \beta\left\|x_{n+1}-x_{n}\right\| \\
& +\left|\alpha_{n}-\alpha_{n+1}\right|\left(\left\|B S_{n+1} y_{n}\right\|+\left\|\gamma f\left(x_{n}\right)\right\|\right)+\left\|S_{n+1} y_{n}-S_{n} y_{n}\right\| \\
\leq & \left(1-\alpha_{n+1} \bar{\gamma}\right)\left\|u_{n+1}-u_{n}\right\|+\alpha_{n+1} \gamma \beta\left\|x_{n+1}-x_{n}\right\|+\left|\alpha_{n}-\alpha_{n+1}\right| M \\
& +\sup \left\{\left\|S_{n+1} z-S_{n} z\right\|: z \in\left\{y_{n}\right\}\right\},
\end{aligned}
$$

where $M:=\sup \left\{\max \left\{\left\|B S_{n+1} y_{n}\right\|,\left\|\gamma f\left(x_{n}\right)\right\|\right\}: n \geq 0\right\}<\infty$. On the other hand, we note that

$$
\begin{gathered}
F\left(u_{n}, y\right)+\frac{1}{r_{n}}\left\langle y-u_{n}, u_{n}-x_{n}\right\rangle \geq 0, \quad \forall y \in H, \\
F\left(u_{n+1}, y\right)+\frac{1}{r_{n+1}}\left\langle y-u_{n+1}, u_{n+1}-x_{n+1}\right\rangle \geq 0, \quad \forall y \in H .
\end{gathered}
$$

Putting $y=u_{n+1}$ in (3.29) and $y=u_{n}$ in (3.30), $F\left(u_{n}, u_{n+1}\right)+\left(1 / r_{n}\right)\left\langle u_{n+1}-u_{n}, u_{n}-x_{n}\right\rangle \geq 0$ and $F\left(u_{n+1}, u_{n}\right)+\left(1 / r_{n+1}\right)\left\langle u_{n}-u_{n+1}, u_{n+1}-x_{n+1}\right\rangle \geq 0$. By (A2), we have

$$
\left\langle u_{n+1}-u_{n}, \frac{u_{n}-x_{n}}{r_{n}}-\frac{u_{n+1}-x_{n+1}}{r_{n+1}}\right\rangle \geq 0
$$

and hence

$$
\left\langle u_{n+1}-u_{n}, u_{n}-u_{n+1}+u_{n+1}-x_{n}-\frac{r_{n}}{r_{n+1}}\left(u_{n+1}-x_{n+1}\right)\right\rangle \geq 0
$$


Since $\liminf _{n \rightarrow \infty} r_{n}>0$, we assume that there exists a real number $b$ such that $r_{n}>b>0$ for all $n \in \mathbb{N}$. Thus, we have

$$
\begin{aligned}
\left\|u_{n+1}-u_{n}\right\|^{2} & \leq\left\langle u_{n+1}-u_{n}, x_{n+1}-x_{n}+\left(1-\frac{r_{n}}{r_{n+1}}\right)\left(u_{n+1}-x_{n+1}\right)\right\rangle \\
& \leq\left\|u_{n+1}-u_{n}\right\|\left\{\left\|x_{n+1}-x_{n}\right\|+\left|1-\frac{r_{n}}{r_{n+1}}\right|\left\|u_{n+1}-x_{n+1}\right\|\right\},
\end{aligned}
$$

and hence

$$
\begin{aligned}
\left\|u_{n+1}-u_{n}\right\| & \leq\left\|x_{n+1}-x_{n}\right\|+\frac{1}{r_{n+1}}\left|r_{n+1}-r_{n}\right|\left\|u_{n+1}-x_{n+1}\right\| \\
& \leq\left\|x_{n+1}-x_{n}\right\|+\frac{1}{b}\left|r_{n+1}-r_{n}\right| L
\end{aligned}
$$

where $L=\sup \left\{\left\|u_{n}-x_{n}\right\|: n \in \mathbb{N}\right\}$. From (3.28), we have

$$
\begin{aligned}
\left\|x_{n+2}-x_{n+1}\right\| \leq & \left(1-\alpha_{n+1} \bar{\gamma}\right)\left(\left\|x_{n+1}-x_{n}\right\|+\frac{1}{b}\left|r_{n+1}-r_{n}\right| L\right)+\alpha_{n+1} \gamma \beta\left\|x_{n+1}-x_{n}\right\| \\
& +\left|\alpha_{n}-\alpha_{n+1}\right| M+\sup \left\{\left\|S_{n+1} z-S_{n} z\right\|: z \in\left\{y_{n}\right\}\right\} \\
= & \left(1-\alpha_{n+1} \bar{\gamma}+\alpha_{n+1} \gamma \beta\right)\left\|x_{n+1}-x_{n}\right\|+\frac{\left(1-\alpha_{n+1} \bar{\gamma}\right)}{b}\left|r_{n+1}-r_{n}\right| L \\
& +\left|\alpha_{n}-\alpha_{n+1}\right| M+\sup \left\{\left\|S_{n+1} z-S_{n} z\right\|: z \in\left\{y_{n}\right\}\right\} \\
\leq & \left(1-\alpha_{n+1}(\bar{\gamma}-\gamma \beta)\right)\left\|x_{n+1}-x_{n}\right\|+\frac{L}{b}\left|r_{n+1}-r_{n}\right|+\left|\alpha_{n}-\alpha_{n+1}\right| M \\
& +\sup \left\{\left\|S_{n+1} z-S_{n} z\right\|: z \in\left\{y_{n}\right\}\right\} .
\end{aligned}
$$

Since $\left\{y_{n}\right\}$ is bounded, it follows that $\sum_{n=1}^{\infty} \sup \left\{\left\|S_{n+1} z-S_{n} z\right\|: z \in\left\{y_{n}\right\}\right\}<\infty$. Hence, by Lemma 2.3, we have $\left\|x_{n+1}-x_{n}\right\| \rightarrow \infty$ as $n \rightarrow \infty$. From (3.34) and $\left|r_{n+1}-r_{n}\right| \rightarrow 0$, we have $\lim _{n \rightarrow \infty}\left\|u_{n+1}-u_{n}\right\|=0$. Moreover, we have from (3.27) that $\lim _{n \rightarrow \infty}\left\|y_{n+1}-y_{n}\right\|=0$.

We note from (3.23) that $x_{n}=\alpha_{n-1} \gamma f\left(x_{n-1}\right)+\left(1-\alpha_{n-1} B\right) S_{n-1} y_{n-1}$. Then, we have

$$
\begin{aligned}
\left\|x_{n}-S_{n} y_{n}\right\| \leq & \left\|x_{n}-S_{n-1} y_{n-1}\right\|+\left\|S_{n-1} y_{n-1}-S_{n-1} y_{n}\right\|+\left\|S_{n-1} y_{n}-S_{n} y_{n}\right\| \\
\leq & \alpha_{n-1}\left\|\gamma f\left(x_{n-1}\right)-B S_{n-1} y_{n-1}\right\|+\left\|y_{n-1}-y_{n}\right\| \\
& +\sup \left\{\left\|S_{n-1} z-S_{n} z\right\|: z \in\left\{y_{n}\right\}\right\} .
\end{aligned}
$$

Since $\alpha_{n} \rightarrow 0,\left\|y_{n-1}-y_{n}\right\| \rightarrow 0$ and $\sup \left\{\left\|S_{n-1} z-S_{n} z\right\|: z \in\left\{y_{n}\right\}\right\} \rightarrow 0$, we get $\left\|x_{n}-S_{n} y_{n}\right\| \rightarrow$ 0 . From the proof of Theorem 3.1, we have

$$
\left\|u_{n}-v\right\|^{2} \leq\left\|x_{n}-v\right\|^{2}-\left\|x_{n}-u_{n}\right\|^{2},
$$


for all $v \in \Omega$. Therefore, we have

$$
\begin{aligned}
\left\|x_{n+1}-v\right\|^{2}= & \left\|\alpha_{n} \gamma f\left(x_{n}\right)+\left(I-\alpha_{n} B\right) S_{n} y_{n}-v\right\|^{2} \\
= & \left\|\left(I-\alpha_{n} B\right)\left(S_{n} y_{n}-v\right)+\alpha_{n}\left(\gamma f\left(x_{n}\right)-B v\right)\right\|^{2} \\
\leq & \left(1-\alpha_{n} \bar{\gamma}\right)^{2}\left\|S_{n} y_{n}-v\right\|^{2}+2 \alpha_{n}\left\langle\gamma f\left(x_{n}\right)-B v, x_{n}-v\right\rangle \\
\leq & \left(1-\alpha_{n} \bar{\gamma}\right)^{2}\left\|y_{n}-v\right\|^{2}+2 \alpha_{n} \gamma\left\langle f\left(x_{n}\right)-f(v), x_{n}-v\right\rangle+2 \alpha_{n}\left\langle\gamma f(v)-B v, x_{n}-v\right\rangle \\
\leq & \left(1-\alpha_{n} \bar{\gamma}\right)^{2}\left\|u_{n}-v\right\|^{2}+2 \alpha_{n} \gamma\left\langle f\left(x_{n}\right)-f(v), x_{n}-v\right\rangle+2 \alpha_{n}\left\langle\gamma f(v)-B v, x_{n}-v\right\rangle \\
\leq & \left(1-\alpha_{n} \bar{\gamma}\right)^{2}\left\{\left\|x_{n}-v\right\|^{2}-\left\|x_{n}-u_{n}\right\|^{2}\right\}+2 \alpha_{n} \gamma \beta\left\|x_{n}-v\right\|^{2} \\
& +2 \alpha_{n}\|\gamma f(v)-B v\|\left\|x_{n}-v\right\| \\
= & \left(1-2 \alpha_{n}(\bar{\gamma}-\gamma \beta)+\left(\alpha_{n} \bar{\gamma}\right)^{2}\right)\left\|x_{n}-v\right\|^{2}-\left(1-\alpha_{n} \bar{\gamma}\right)^{2}\left\|x_{n}-u_{n}\right\|^{2} \\
& +2 \alpha_{n}\|\gamma f(v)-B v\|\left\|x_{n}-v\right\| \\
\leq & \left\|x_{n}-v\right\|^{2}+\alpha_{n} \bar{\gamma}^{2}\left\|x_{n}-v\right\|^{2}-\left(1-\alpha_{n} \bar{\gamma}\right)^{2}\left\|x_{n}-u_{n}\right\|^{2}+2 \alpha_{n}\|\gamma f(v)-B v\|\left\|x_{n}-v\right\|
\end{aligned}
$$

and hence

$$
\begin{aligned}
\left(1-\alpha_{n} \bar{\gamma}\right)^{2}\left\|x_{n}-u_{n}\right\|^{2} \leq & \alpha_{n} \bar{\gamma}^{2}\left\|x_{n}-v\right\|^{2}+2 \alpha_{n}\|\gamma f(v)-B v\|\left\|x_{n}-v\right\|+\left\|x_{n}-v\right\|^{2}-\left\|x_{n+1}-v\right\|^{2} \\
\leq & \alpha_{n} \bar{\gamma}^{2}\left\|x_{n}-v\right\|^{2}+2 \alpha_{n}\|\gamma f(v)-B v\|\left\|x_{n}-v\right\| \\
& +\left\|x_{n}-x_{n+1}\right\|\left(\left\|x_{n}-v\right\|+\left\|x_{n+1}-v\right\|\right) .
\end{aligned}
$$

Since $\left\{x_{n}\right\}$ is bounded, $\alpha_{n} \rightarrow 0$ and $\left\|x_{n}-x_{n+1}\right\| \rightarrow 0$, it follows that $\left\|x_{n}-u_{n}\right\| \rightarrow 0$ as $n \rightarrow \infty$.

Put $M=\sup _{n \geq 1}\left\{\|\gamma f(v)-B v\|\left\|x_{n}-v\right\|\right\}$. It follows from (3.38), the nonexpansive of $J_{M, \lambda}$ and the inverse strongly monotonicity of $A$ that

$$
\begin{aligned}
\left\|x_{n+1}-v\right\|^{2} \leq & \left(1-\alpha_{n} \bar{\gamma}\right)^{2}\left\|y_{n}-v\right\|^{2}+2 \alpha_{n} \gamma \beta\left\|x_{n}-v\right\|^{2}+2 \alpha_{n} M \\
\leq & \left(1-\alpha_{n} \bar{\gamma}\right)^{2}\left\|\left(u_{n}-\lambda A u_{n}\right)-(v-\lambda A v)\right\|^{2}+2 \alpha_{n} \gamma \beta\left\|x_{n}-v\right\|^{2}+2 \alpha_{n} M \\
\leq & \left(1-\alpha_{n} \bar{\gamma}\right)^{2}\left\{\left\|u_{n}-v\right\|^{2}+\lambda(\lambda-2 \alpha)\left\|A u_{n}-A v\right\|^{2}\right\}+2 \alpha_{n} \gamma \beta\left\|x_{n}-v\right\|^{2}+2 \alpha_{n} M \\
\leq & \left(1-\alpha_{n} \bar{\gamma}\right)^{2}\left\|x_{n}-v\right\|^{2}+\left(1-\alpha_{n} \bar{\gamma}\right)^{2} \lambda(\lambda-2 \alpha)\left\|A u_{n}-A v\right\|^{2} \\
& +2 \alpha_{n} \gamma \beta\left\|x_{n}-v\right\|^{2}+2 \alpha_{n} M \\
= & \left(1-2 \alpha_{n}(\bar{\gamma}-\gamma \beta)+\left(\alpha_{n} \bar{\gamma}\right)^{2}\right)\left\|x_{n}-v\right\|^{2}+\left(1-\alpha_{n} \bar{\gamma}\right)^{2} \lambda(\lambda-2 \alpha)\left\|A u_{n}-A v\right\|^{2}+2 \alpha_{n} M \\
\leq & \left\|x_{n}-v\right\|^{2}+\alpha_{n} \bar{\gamma}^{2}\left\|x_{n}-v\right\|^{2}+\left(1-\alpha_{n} \bar{\gamma}\right)^{2} \lambda(\lambda-2 \alpha)\left\|A u_{n}-A v\right\|^{2}+2 \alpha_{n} M .
\end{aligned}
$$


This implies that

$$
\begin{aligned}
\left(1-\alpha_{n} \bar{\gamma}\right)^{2} \lambda(2 \alpha-\lambda)\left\|A u_{n}-A v\right\|^{2} & \leq \alpha_{n} \bar{\gamma}^{2}\left\|x_{n}-v\right\|^{2}+2 \alpha_{n} M+\left\|x_{n}-v\right\|^{2}-\left\|x_{n+1}-v\right\|^{2} \\
& \leq \alpha_{n} \bar{\gamma}^{2}\left\|x_{n}-v\right\|^{2}+2 \alpha_{n} M+\left\|x_{n}-x_{n+1}\right\|\left(\left\|x_{n}-v\right\|+\left\|x_{n+1}-v\right\|\right)
\end{aligned}
$$

Since $\alpha_{n} \rightarrow 0$ and $\left\|x_{n}-x_{n+1}\right\| \rightarrow 0$, we have $\left\|A u_{n}-A v\right\| \rightarrow 0$ as $n \rightarrow \infty$.From (3.5), (3.15) and (3.38), we have

$$
\begin{aligned}
\left\|x_{n+1}-v\right\|^{2} \leq & \left(1-\alpha_{n} \bar{\gamma}\right)^{2}\left\|y_{n}-v\right\|^{2}+2 \alpha_{n} \gamma \beta\left\|x_{n}-v\right\|^{2}+2 \alpha_{n} M \\
\leq & \left(1-\alpha_{n} \bar{\gamma}\right)^{2}\left\{\left\|u_{n}-v\right\|^{2}-\left\|u_{n}-y_{n}\right\|^{2}+2 \lambda\left\langle u_{n}-y_{n}, A u_{n}-A v\right\rangle-\lambda^{2}\left\|A u_{n}-A v\right\|^{2}\right\} \\
& +2 \alpha_{n} \gamma \beta\left\|x_{n}-v\right\|^{2}+2 \alpha_{n} M \\
\leq & \left(1-\alpha_{n} \bar{\gamma}\right)^{2}\left\|x_{n}-v\right\|^{2}-\left(1-\alpha_{n} \bar{\gamma}\right)^{2}\left\|u_{n}-y_{n}\right\|^{2}+2\left(1-\alpha_{n} \bar{\gamma}\right)^{2} \lambda\left\langle u_{n}-y_{n}, A u_{n}-A v\right\rangle \\
& -\left(1-\alpha_{n} \bar{\gamma}\right)^{2} \lambda^{2}\left\|A u_{n}-A v\right\|^{2}+2 \alpha_{n} \gamma \beta\left\|x_{n}-v\right\|^{2}+2 \alpha_{n} M \\
= & \left(1-2 \alpha_{n}(\bar{\gamma}-\gamma \beta)+\left(\alpha_{n} \bar{\gamma}\right)^{2}\right)\left\|x_{n}-v\right\|^{2}-\left(1-\alpha_{n} \bar{\gamma}\right)^{2}\left\|u_{n}-y_{n}\right\|^{2} \\
& +2\left(1-\alpha_{n} \bar{\gamma}\right)^{2} \lambda\left\langle u_{n}-y_{n}, A u_{n}-A v\right\rangle-\left(1-\alpha_{n} \bar{\gamma}\right)^{2} \lambda^{2}\left\|A u_{n}-A v\right\|^{2}+2 \alpha_{n} M \\
\leq & \left\|x_{n}-v\right\|^{2}+\alpha_{n} \bar{\gamma}^{2}\left\|x_{n}-v\right\|^{2}-\left(1-\alpha_{n} \bar{\gamma}\right)^{2}\left\|u_{n}-y_{n}\right\|^{2} \\
& +2\left(1-\alpha_{n} \bar{\gamma}\right)^{2} \lambda\left\langle u_{n}-y_{n}, A u_{n}-A v\right\rangle-\left(1-\alpha_{n} \bar{\gamma}\right)^{2} \lambda^{2}\left\|A u_{n}-A v\right\|^{2}+2 \alpha_{n} M .
\end{aligned}
$$

Thus, we obtain

$$
\begin{aligned}
\left(1-\alpha_{n} \bar{\gamma}\right)^{2}\left\|u_{n}-y_{n}\right\|^{2} \leq & \alpha_{n} \bar{\gamma}^{2}\left\|x_{n}-v\right\|^{2}+2\left(1-\alpha_{n} \bar{\gamma}\right)^{2} \lambda\left\langle u_{n}-y_{n}, A u_{n}-A v\right\rangle \\
& -\left(1-\alpha_{n} \bar{\gamma}\right)^{2} \lambda^{2}\left\|A u_{n}-A v\right\|^{2}+2 \alpha_{n} M+\left\|x_{n}-v\right\|^{2}-\left\|x_{n+1}-v\right\|^{2} \\
\leq & \alpha_{n} \bar{\gamma}^{2}\left\|x_{n}-v\right\|^{2}+2\left(1-\alpha_{n} \bar{\gamma}\right)^{2} \lambda\left\langle u_{n}-y_{n}, A u_{n}-A v\right\rangle \\
& -\left(1-\alpha_{n} \bar{\gamma}\right)^{2} \lambda^{2}\left\|A u_{n}-A v\right\|^{2}+2 \alpha_{n} M+\left\|x_{n}-x_{n+1}\right\|\left(\left\|x_{n}-v\right\|+\left\|x_{n+1}-v\right\|\right)
\end{aligned}
$$

Since $\alpha_{n} \rightarrow 0,\left\|A u_{n}-A v\right\| \rightarrow 0$ and $\left\|x_{n}-x_{n+1}\right\| \rightarrow 0$, we have $\left\|u_{n}-y_{n}\right\| \rightarrow 0$ as $n \rightarrow \infty$. It follows from the inequality $\left\|y_{n}-S_{n} y_{n}\right\| \leq\left\|y_{n}-u_{n}\right\|+\left\|u_{n}-x_{n}\right\|+\left\|x_{n}-S_{n} y_{n}\right\|$ that $\left\|y_{n}-S_{n} y_{n}\right\| \rightarrow 0$ as $n \rightarrow \infty$. Moreover, we note that $\left\|x_{n}-y_{n}\right\| \leq\left\|x_{n}-u_{n}\right\|+\left\|u_{n}-y_{n}\right\| \rightarrow 0$ as $n \rightarrow \infty$. Since

$$
\begin{aligned}
\left\|S y_{n}-y_{n}\right\| & \leq\left\|S y_{n}-S_{n} y_{n}\right\|+\left\|S_{n} y_{n}-y_{n}\right\| \\
& \leq \sup \left\{\left\|S z-S_{n} z\right\|: z \in\left\{y_{n}\right\}\right\}+\left\|S_{n} y_{n}-y_{n}\right\|
\end{aligned}
$$


for all $n \in \mathbb{N}$, it follows that $\lim _{n \rightarrow \infty}\left\|S y_{n}-y_{n}\right\|=0$. Next, we show that

$$
\limsup _{n \rightarrow \infty}\left\langle(B-\gamma f) z, z-x_{n}\right\rangle \leq 0
$$

where $z=P_{\Omega}(I-B+\gamma f)(z)$ is a unique solution of the variational inequality (3.4). To show this inequality, we choose a subsequence $\left\{x_{n_{i}}\right\}$ of $\left\{x_{n}\right\}$ such that

$$
\lim _{i \rightarrow \infty}\left\langle(B-\gamma f) z, z-x_{n_{i}}\right\rangle=\limsup _{n \rightarrow \infty}\left\langle(B-\gamma f) z, z-x_{n}\right\rangle .
$$

Since $\left\{u_{n_{i}}\right\}$ is bounded, there exists a subsequence $\left\{u_{n_{i j}}\right\}$ of $\left\{u_{n_{i}}\right\}$ which converges weakly to $w$. Without loss of generality, we can assume that $u_{n_{i}} \rightarrow w$. From $\left\|u_{n}-y_{n}\right\| \rightarrow 0$, we obtain $y_{n_{i}} w$. Let us show $w \in E P(F)$. It follows by (3.23) and (A2) that

$$
\frac{1}{r_{n}}\left\langle y-u_{n}, u_{n}-x_{n}\right\rangle \geq F\left(y, u_{n}\right)
$$

and hence

$$
\left\langle y-u_{n_{i}}, \frac{u_{n_{i}}-x_{n_{i}}}{r_{n_{i}}}\right\rangle \geq F\left(y, u_{n_{i}}\right) .
$$

Since $\left(u_{n_{i}}-x_{n_{i}}\right) / r_{n_{i}} \rightarrow 0$ and $u_{n_{i}} \rightarrow w$, it follows by (A4) that $0 \geq F(y, w)$ for all $y \in H$. For $t$ with $0<t \leq 1$ and $y \in H$, let $y_{t}=t y+(1-t) w$. Since $y \in H$ and $w \in H$, we have $y_{t} \in H$ and hence $F\left(y_{t}, w\right) \leq 0$. So, from (A1) and (A4) we have

$$
0=F\left(y_{t}, y_{t}\right) \leq t F\left(y_{t}, y\right)+(1-t) F\left(y_{t}, w\right) \leq t F\left(y_{t}, y\right)
$$

and hence $0 \leq F\left(y_{t}, y\right)$. From (A3), we have $0 \leq F(w, y)$ for all $y \in H$ and hence $w \in E P(F)$. By the same argument as in proof of Theorem 3.1, we have $w \in F(S) \cap I(A, M)$ and hence $w \in \Omega$. This implies that

$$
\limsup _{n \rightarrow \infty}\left\langle(B-\gamma f) z, z-x_{n}\right\rangle=\lim _{i \rightarrow \infty}\left\langle(B-\gamma f) z, z-x_{n_{i}}\right\rangle=\langle(B-\gamma f) z, z-w\rangle \leq 0
$$


Finally we prove that $x_{n} \rightarrow z$. From (3.23), we have

$$
\begin{aligned}
\left\|x_{n+1}-z\right\|^{2} & =\left\|\alpha_{n} \gamma f\left(x_{n}\right)+\left(I-\alpha_{n} B\right) S_{n} y_{n}-z\right\|^{2} \\
& =\left\|\alpha_{n}\left(\gamma f\left(x_{n}\right)-B z\right)+\left(I-\alpha_{n} B\right)\left(S_{n} y_{n}-z\right)\right\|^{2} \\
& \leq\left\|\left(I-\alpha_{n} B\right)\left(S_{n} y_{n}-z\right)\right\|^{2}+2 \alpha_{n}\left\langle\gamma f\left(x_{n}\right)-B z, x_{n+1}-z\right\rangle \\
& \leq\left(1-\alpha_{n} \bar{\gamma}\right)^{2}\left\|y_{n}-z\right\|^{2}+2 \alpha_{n} \gamma\left\langle f\left(x_{n}\right)-f(z), x_{n+1}-z\right\rangle+2 \alpha_{n}\left\langle\gamma f(z)-B z, x_{n+1}-z\right\rangle \\
& \leq\left(1-\alpha_{n} \bar{\gamma}\right)^{2}\left\|x_{n}-z\right\|^{2}+2 \alpha_{n} \gamma \beta\left\|x_{n}-z\right\|\left\|x_{n+1}-z\right\|+2 \alpha_{n}\left\langle\gamma f(z)-B z, x_{n+1}-z\right\rangle \\
& \leq\left(1-\alpha_{n} \bar{\gamma}\right)^{2}\left\|x_{n}-z\right\|^{2}+\alpha_{n} \gamma \beta\left(\left\|x_{n}-z\right\|^{2}+\left\|x_{n+1}-z\right\|^{2}\right)+2 \alpha_{n}\left\langle\gamma f(z)-B z, x_{n+1}-z\right\rangle .
\end{aligned}
$$

This implies that

$$
\begin{aligned}
\left\|x_{n+1}-z\right\|^{2} \leq & \frac{1-2 \alpha_{n} \bar{\gamma}+\left(\alpha_{n} \bar{\gamma}\right)^{2}+\alpha_{n} \gamma \beta}{1-\alpha_{n} \gamma \beta}\left\|x_{n}-z\right\|^{2}+\frac{2 \alpha_{n}}{1-\alpha_{n} \gamma \beta}\left\langle\gamma f(z)-A z, x_{n+1}-z\right\rangle \\
= & {\left[1-\frac{2(\bar{\gamma}-\gamma \beta) \alpha_{n}}{1-\alpha_{n} \gamma \beta}\right]\left\|x_{n}-z\right\|^{2}+\frac{\left(\alpha_{n} \bar{\gamma}\right)^{2}}{1-\alpha_{n} \gamma \beta}\left\|x_{n}-z\right\|^{2} } \\
& +\frac{2 \alpha_{n}}{1-\alpha_{n} \gamma \beta}\left\langle\gamma f(z)-A z, x_{n+1}-z\right\rangle \\
\leq & \left(1-\gamma_{n}\right)\left\|x_{n}-z\right\|^{2}+\delta_{n},
\end{aligned}
$$

where $\gamma_{n}:=\left(2 \alpha_{n}(\bar{\gamma}-\gamma \beta)\right) /\left(1-\alpha_{n} \gamma \beta\right)$ and $\delta_{n}:=\alpha_{n} /\left(1-\alpha_{n} \gamma \beta\right)\left\{\alpha_{n} \bar{\gamma}^{2}\left\|x_{n}-z\right\|^{2}+2\langle\gamma f(z)-\right.$ $\left.\left.A z, x_{n+1}-z\right\rangle\right\}$. It easily verified that $\gamma_{n} \rightarrow 0, \sum_{n=1}^{\infty} \gamma_{n}=\infty$ and $\lim \sup _{n \rightarrow \infty} \delta n / \gamma_{n} \leq 0$. Hence, by Lemma 2.1, the sequence $\left\{x_{n}\right\}$ converges strongly to $z$.

As in [10, Theorem 4.1], we can generate a sequence $\left\{S_{n}\right\}$ of nonexpansive mappings satisfying condition $\sum_{n=1}^{\infty} \sup \left\{\left\|S_{n+1} z-S_{n} z\right\|: z \in K\right\}<\infty$. for any bounded $K$ of $H$ by using convex combination of a general sequence $\left\{T_{k}\right\}$ of nonexpansive mappings with a common fixed point.

Corollary 3.3. Let $H$ be a real Hilbert space, let $F$ be a bifunction from $H \times H \rightarrow \mathbb{R}$ satisfying (A1)-(A4) and let $A: H \rightarrow H$ be an $\alpha$-inverse-strongly monotone mapping, $M: H \rightarrow 2^{H}$ be a maximal monotone mapping. Let $f$ be a contraction of $H$ into itself with a constant $\beta \in(0,1)$ and let $B$ be a strongly bounded linear operator on $H$ with coefficient $\bar{\gamma}>0$ and $0<\gamma<\bar{\gamma} / \beta$. Let $\left\{\beta_{n}^{k}\right\}$ be a family of nonnegative numbers with indices $n, k \in \mathbb{N}$ with $k \leq n$ such that

(i) $\sum_{k=1} \beta_{n}^{k}=1$, for all $n \in \mathbb{N}$;

(ii) $\lim _{n \rightarrow \infty} \beta_{n}^{k}>0$, for every $k \in \mathbb{N}$;

(iii) $\sum_{n=1}^{\infty} \sum_{k=1}^{n}\left|\beta_{n+1}^{k}-\beta_{n}^{k}\right|<\infty$. 
Let $\left\{T_{k}\right\}$ be a sequence of nonexpansive mappings on $H$ with $\Omega:=F\left(\bigcap_{k=1}^{\infty} F\left(T_{k}\right)\right) \cap E P(F) \cap$ $I(A, M) \neq \varnothing$ and let $\left\{x_{n}\right\},\left\{y_{n}\right\}$ and $\left\{u_{n}\right\}$ be sequences generated by $x_{1} \in H$ and

$$
\begin{gathered}
F\left(u_{n}, y\right)+\frac{1}{r_{n}}\left\langle y-u_{n}, u_{n}-x_{n}\right\rangle \geq 0, \quad \forall y \in H \\
x_{n+1}=\alpha_{n} \gamma f\left(x_{n}\right)+\left(I-\alpha_{n} B\right) \sum_{k=1}^{n} \beta_{n}^{k} T_{k} y_{n} \\
y_{n}=J_{M, \lambda}\left(u_{n}-\lambda A u_{n}\right) \quad \forall n \geq 0
\end{gathered}
$$

for all $n \in \mathbb{N}$, where $\lambda \in(0,2 \alpha],\left\{\alpha_{n}\right\} \subset[0,1]$ and $\left\{r_{n}\right\} \subset(0, \infty)$ satisfy

$$
\begin{gathered}
\lim _{n \rightarrow \infty} \alpha_{n}=0, \quad \sum_{n=1}^{\infty} \alpha_{n}=\infty, \quad \sum_{n=1}^{\infty}\left|\alpha_{n+1}-\alpha_{n}\right|<\infty, \\
\liminf _{n \rightarrow \infty} r_{n}>0, \quad \sum_{n=1}^{\infty}\left|r_{n+1}-r_{n}\right|<\infty .
\end{gathered}
$$

Then, $\left\{x_{n}\right\},\left\{y_{n}\right\}$ and $\left\{u_{n}\right\}$ converges strongly to $z$ in $\Omega$ which solves the variational inequality:

$$
\langle(B-\gamma f) z, z-x\rangle \geq 0, \quad x \in \Omega
$$

If $S_{n}=S, B \equiv I$ and $\gamma=1$ in Theorem 3.2, we obtain the following corollary.

Corollary 3.4 (see Peng et al. [9]). Let $H$ be a real Hilbert space, let $F$ be a bifunction from $C \times C \rightarrow$ $\mathbb{R}$ satisfying (A1)-(A4) and let $S$ be a nonexpansive mapping on $H$. Let $A: H \rightarrow H$ be an $\alpha$ inverse-strongly monotone mapping, $M: H \rightarrow 2^{H}$ be a maximal monotone mapping such that $\Omega:=F(S) \cap E P(F) \cap I(A, M) \neq \varnothing$. Let $f$ be a contraction of $H$ into itself with a constant $\beta \in(0,1)$. Let $\left\{x_{n}\right\},\left\{y_{n}\right\}$ and $\left\{u_{n}\right\}$ be sequences generated by $x_{1} \in H$ and

$$
\begin{gathered}
F\left(u_{n}, y\right)+\frac{1}{r_{n}}\left\langle y-u_{n}, u_{n}-x_{n}\right\rangle \geq 0, \quad \forall y \in H \\
x_{n+1}=\alpha_{n} f\left(x_{n}\right)+\left(I-\alpha_{n}\right) S y_{n} \\
y_{n}=J_{M, \lambda}\left(u_{n}-\lambda A u_{n}\right) \quad \forall n \geq 0
\end{gathered}
$$

for all $n \in \mathbb{N}$, where $\lambda \in(0,2 \alpha],\left\{\alpha_{n}\right\} \subset[0,1]$ and $\left\{r_{n}\right\} \subset(0, \infty)$ satisfy

$$
\begin{gathered}
\lim _{n \rightarrow \infty} \alpha_{n}=0, \quad \sum_{n=1}^{\infty} \alpha_{n}=\infty, \quad \sum_{n=1}^{\infty}\left|\alpha_{n+1}-\alpha_{n}\right|<\infty, \\
\liminf _{n \rightarrow \infty} r_{n}>0, \quad \sum_{n=1}^{\infty}\left|r_{n+1}-r_{n}\right|<\infty .
\end{gathered}
$$

Then, $\left\{x_{n}\right\},\left\{y_{n}\right\}$ and $\left\{u_{n}\right\}$ converges strongly to $z \in \Omega$, where $z=P_{\Omega} f(z)$. 
If $S_{n}=S, A \equiv 0$ and $M \equiv 0$ in Theorem 3.2, we obtain the following corollary.

Corollary 3.5 (see S. Plubtieng and R. Punpaeng [6]). Let $H$ be a real Hilbert space, let $F$ be a bifunction from $H \times H \rightarrow \mathbb{R}$ satisfying (A1)-(A4) and let $S$ be a nonexpansive mapping on $H$ such that $\Omega:=F(S) \cap E P(F) \neq \varnothing$. Let $f$ be a contraction of $H$ into itself with a constant $\beta \in(0,1)$ and let $B$ be a strongly bounded linear operator on $H$ with coefficient $\bar{\gamma}>0$ and $0<\gamma<\bar{\gamma} / \beta$. Let $\left\{x_{n}\right\},\left\{u_{n}\right\}$ and be sequences generated by $x_{1} \in H$ and

$$
\begin{gathered}
F\left(u_{n}, y\right)+\frac{1}{r_{n}}\left\langle y-u_{n}, u_{n}-x_{n}\right\rangle \geq 0, \quad \forall y \in H \\
x_{n+1}=\alpha_{n} \gamma f\left(x_{n}\right)+\left(I-\alpha_{n} B\right) S u_{n},
\end{gathered}
$$

for all $n \in \mathbb{N}$, where $\left\{\alpha_{n}\right\} \subset[0,1]$ and $\left\{r_{n}\right\} \subset(0, \infty)$ satisfy

$$
\begin{gathered}
\lim _{n \rightarrow \infty} \alpha_{n}=0, \quad \sum_{n=1}^{\infty} \alpha_{n}=\infty, \quad \sum_{n=1}^{\infty}\left|\alpha_{n+1}-\alpha_{n}\right|<\infty, \\
\liminf _{n \rightarrow \infty} r_{n}>0, \quad \sum_{n=1}^{\infty}\left|r_{n+1}-r_{n}\right|<\infty .
\end{gathered}
$$

Then, $\left\{x_{n}\right\}$ and $\left\{u_{n}\right\}$ converges strongly to a point $z$ in $\Omega$ which solves the variational inequality:

$$
\langle(B-\gamma f) z, z-x\rangle \geq 0, \quad x \in \Omega \text {. }
$$

\section{Acknowledgments}

The first author thank the National Research Council of Thailand to Naresuan University, 2009 for the financial support. Moreover, the second author would like to thank the "National Centre of Excellence in Mathematics", PERDO, under the Commission on Higher Education, Ministry of Education, Thailand.

\section{References}

[1] P. L. Combettes and S. A. Hirstoaga, "Equilibrium programming in Hilbert spaces," Journal of Nonlinear and Convex Analysis, vol. 6, no. 1, pp. 117-136, 2005.

[2] L. C. Zeng, S. Schaible, and J. C. Yao, "Iterative algorithm for generalized set-valued strongly nonlinear mixed variational-like inequalities," Journal of Optimization Theory and Applications, vol. 124, no. 3, pp. 725-738, 2005.

[3] S. Plubtieng and R. Punpaeng, "A new iterative method for equilibrium problems and fixed point problems of nonexpansive mappings and monotone mappings," Applied Mathematics and Computation, vol. 197, no. 2, pp. 548-558, 2008.

[4] S.-S. Chang, H. W. Joseph Lee, and C. K. Chan, "A new method for solving equilibrium problem fixed point problem with application to optimization," Nonlinear Analysis: Theory, Methods $\mathcal{E}$ Applications, vol. 70, pp. 3307-3319, 2009.

[5] V. Colao, G. Marino, and H.-K. Xu, "An iterative method for finding common solutions of equilibrium and fixed point problems," Journal of Mathematical Analysis and Applications, vol. 344, no. 1, pp. 340352, 2008. 
[6] S. Plubtieng and R. Punpaeng, "A general iterative method for equilibrium problems and fixed point problems in Hilbert spaces," Journal of Mathematical Analysis and Applications, vol. 336, no. 1, pp. 455$469,2007$.

[7] R. T. Rockafellar, "Monotone operators and the proximal point algorithm," SIAM Journal on Control and Optimization, vol. 14, no. 5, pp. 877-898, 1976.

[8] S. Adly, "Perturbed algorithms and sensitivity analysis for a general class of variational inclusions," Journal of Mathematical Analysis and Applications, vol. 201, no. 2, pp. 609-630, 1996.

[9] J.-W. Peng, Y. Wang, D. S. Shyu, and J.-C. Yao, "Common solutions of an iterative scheme for variational inclusions, equilibrium problems, and fixed point problems," Journal of Inequalities and Applications, vol. 2008, Article ID 720371, 15 pages, 2008.

[10] K. Aoyama, Y. Kimura, W. Takahashi, and M. Toyoda, "Approximation of common fixed points of a countable family of nonexpansive mappings in a Banach space," Nonlinear Analysis: Theory, Methods E Applications, vol. 67, no. 8, pp. 2350-2360, 2007.

[11] E. Blum and W. Oettli, "From optimization and variational inequalities to equilibrium problems," The Mathematics Student, vol. 63, no. 1-4, pp. 123-145, 1994.

[12] G. Marino and H.-K. Xu, "A general iterative method for nonexpansive mappings in Hilbert spaces," Journal of Mathematical Analysis and Applications, vol. 318, no. 1, pp. 43-52, 2006.

[13] H.-K. Xu, "Iterative algorithms for nonlinear operators," Journal of the London Mathematical Society, vol. 66, no. 1, pp. 240-256, 2002.

[14] H. Brézis, Opérateurs Maximaux Monotones et Semi-Groupes de Contractions dans les Espaces de Hilbert, North-Holland, Amsterdam, The Netherlands, 1973.

[15] B. Lemaire, "Which fixed point does the iteration method select?" in Recent Advances in Optimization (Trier, 1996), vol. 452 of Lecture Notes in Economics and Mathematical Systems, pp. 154-167, Springer, Berlin, Germany, 1997. 